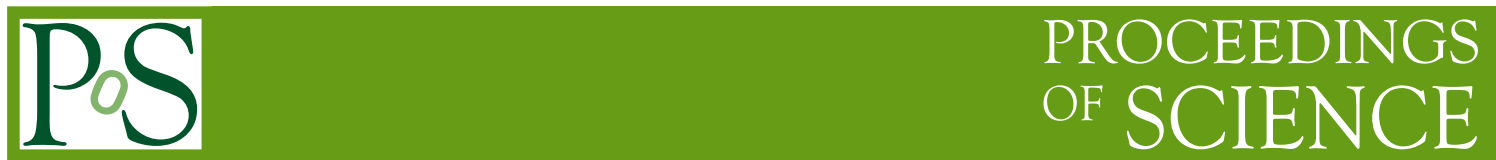

\title{
The Chemical Evolution of Galaxies
}

\author{
H. Jabran Zahid** \\ Harvard-Smithsonian Center for Astrophysics \\ E-mail: hzahidecfa.harvard.edu
}

The gas-phase oxygen abundance, i.e. metallicity, of a galaxy is set by the interplay between star formation and gas flows. Metals are dispersed into the interstellar medium by stellar winds and supernovae. Metals accumulate in the interstellar medium of star-forming galaxies and provide a record of star-formation. However, inflows of unenriched gas into galaxies and metal-rich outflows of gas from galaxies can both reduce the metallicity. Thus, measurements of the metallicity across cosmic time provide important constraints for understanding the cycling of gas through galaxies as they build their stellar mass and evolve. We have measured the chemical evolution of galaxies over the last 10 billion years of cosmic time. These measurements provide a coherent picture of how galaxies enrich as they build their stellar mass. We show that the chemical evolution of star-forming galaxies is very simple. The metallicity of star-forming galaxies at $z<1.5$ only depends on the stellar-to-gas mass ratio. The relation between metallicity and stellar-to-gas mass ratio is an universal relation followed by all galaxies as they evolve.

Frank N. Bash Symposium 2015

18-20 October

The University of Texas at Austin, USA

\footnotetext{
* Speaker.

†Clay Postdoctoral Fellow.
} 


\section{Introduction}

Gas flows and star formation govern the evolution of galaxies. A key diagnostic of gas flows and star formation in galaxies is the amount of heavy elements relative to hydrogen in the interstellar medium (ISM). Heavy elements are produced by massive stars and are dispersed into the ISM by stellar mass loss processes. Therefore, the ISM metal content is closely linked to the stellar mass of a galaxy. The heavy element abundance is measured relative to hydrogen and therefore also depends on the gas content of galaxies. The rate at which stars form is also regulated by the gas content. Understanding the relation between stellar mass, metallicity and star formation rate (SFR) of galaxies and its evolution is fundamental for developing a comprehensive theory of galaxy evolution.

Oxygen is the most abundant heavy element formed in the Universe. Therefore, the abundance of oxygen can be used as a proxy for the production of all heavy elements. The gas-phase oxygen abundance is correlated to the stellar mass in star-forming galaxies. This relation is known as the mass-metallicity (MZ) relation. The MZ relation was first observed in a small sample of nearby galaxies by Lequeux et al. [1]. They showed that galaxy metallicity increases with stellar mass. Subsequently, Tremonti et al. [2] measure the MZ relation of $\sim 50,000$ star-forming galaxies in the Sloan Digital Sky Survey (SDSS). They find a tight MZ relation $(\sim 0.1$ dex scatter) extending over three orders of magnitude in stellar mass.

Several independent approaches suggest that the MZ relation extends down to very low stellar masses. From the examination of $\sim 27$ nearby dwarf irregular galaxies, Lee et al. [3] argue that the MZ relation extends down to $\sim 10^{6} M_{\odot}$. Berg et al. [4] reach similar conclusions from examination of 42 low luminosity galaxies in the local volume. Based on a substantially larger sample, in Zahid et al. [5] we show that the scatter in metallicities increases at lower stellar masses and the distribution appears to follow the observed MZ relation for galaxies with stellar masses $\gtrsim 10^{9} M_{\odot}$. Finally, from stacking analysis, Andrews \& Martini [6] show that the MZ relation is continuous down to $10^{7} M_{\odot}$.

The MZ relation is one of the primary observations for measuring the chemical evolution of galaxies. Savaglio et al. [7] first showed the MZ relation for galaxies outside the local Universe. Many subsequent studies have shown the MZ relation is observed for galaxies out to $z \sim 3[8,9$, $10,11,12,13,14,15,16,17,18,19]$ and perhaps beyond [20,21]. Observations of the MZ relation reveal that the metallicities of galaxies increase with time.

The scatter observed in the MZ relation is correlated with other physical properties of galaxies. These correlations provide clues to the origin of the MZ relation. Ellison et al. [22] first showed that the MZ relation depends on specific SFR and galaxy size. At a fixed stellar mass, galaxies with high specific SFRs or large half-light radii have systematically lower gas-phase metallicities than galaxies with low specific SFRs or small radii.

Several studies have built on the results of Ellison et al. [22]. A relation between stellar mass, metallicity and star formation rate (SFR) is observed in local $[23,24,25,6]$ and high redshift galaxies $[18,19,26]$. At stellar masses $\lesssim 10^{10.5} M_{\odot}$, galaxies with high SFRs typically have lower metallicities and vice versa. Mannucci et al. [23] derive a relation between stellar mass, metallicity and SFR that minimizes the scatter of metallicity in the local galaxy population. They argue that the minimum scatter relation between stellar mass, metallicity and SFR that they derive is independent 
of redshift. They refer to this minimum scatter relation as the fundamental metallicity relation (FMR). For the FMR, the higher SFRs observed in high redshift galaxies account for their lower metallicities. However, the redshift independence of the relation between stellar mass, metallicity and SFR remains tentative $[27,28,29,18,30]$.

Both the SFR and metallicity are dependent on the gas content and the anti-correlation between metallicity and SFR is likely the result of variations in gas content [31,32,33]. Recently, Bothwell et al. [33] present observations suggesting that the FMR derived by Mannucci et al. [23] is the result of a relation between stellar mass, metallicity and gas content. At a fixed stellar mass, galaxies with higher gas fractions will exhibit elevated SFRs and lower metallicity. In the Bothwell et al. [33] interpretation, the SFR acts as a proxy for gas content in the FMR proposed by Mannucci et al. [23]. Bothwell et al. [33] are not able to investigate the redshift dependence of the relation between stellar mass, metallicity and gas content due to lack of measurements of atomic gas in galaxies outside the local Universe.

Gas outflows deplete the heavy element content of galaxies. Typically it is assumed that the wind material has the same metallicity as the ambient ISM. However, the actual wind metallicity relative to the ISM may be greater if it is primarily comprised of supernova ejecta or substantially depressed if a sufficient amount of metal-poor gas is entrained as the wind propagates out of the galaxy. The total amount of metals ejected from the ISM will be proportional to the magnitude and metallicity of the outflowing gas, modulo the amount of metals ejected and subsequently reaccreted. In the absence of direct measurements characterizing these physical processes, we must rely on empirical constraints for the total metal loss in the local galaxy population to infer the properties of outflows and reaccretion.

Uncovering the origin of the MZ relation is crucial for understanding gas flows and star formation in galaxies. It is clear that the MZ relation depends on gas flows and star formation. Still, despite numerous studies of the MZ relation over the last few decades, the physical origin of the $\mathrm{MZ}$ relation remains uncertain. The MZ relation could possibly be the result of metal-rich outflows [34], inefficient star formation in less massive galaxies [35, 36, 37], metal-poor inflows [38], variations in the initial mass function [39] or some combination of these physical processes.

In this contribution, we will show recent measurements of the MZ relation and its evolution with redshift. We will compare these measurements with analytical equations of galactic chemical evolution in order to understand the origin of the MZ relation. We will conclude by arguing that the MZ relation originates from a universal relation between metallicity and the stellar-to-gas mass ratio. For further reading on this topic, we refer the reader to Zahid et al. 2014 [40].

\section{The Mass-Metallicity Relation and its Evolution}

Many mathematical models may quantitatively fit data but not all models are physically interpretable. For example, the $\mathrm{MZ}$ relation can be fit by a polynomial. However, the best fit parameters derived from a polynomial fit can not be straightforwardly interpreted since the model parameters are not related to physical quantities. We propose a new model for fitting the $\mathrm{MZ}$ relation which we can physically interpret. This model is similar in form to the model proposed by Moustakas et al. [41] and used in Zahid et al. [17]. We emphasize that the parameterization adopted in this work is different. Our interpretation of the fit parameters is presented in the following section. We model 
the $\mathrm{MZ}$ relation as

$$
12+\log (O / H)=Z_{o}+\log \left[1-\exp \left(-\left[\frac{M_{*}}{M_{o}}\right]^{\gamma}\right)\right] .
$$

In this model, $Z_{o}$ is the saturation metallicity. It quantifies the asymptotic upper metallicity limit $[41,17] . M_{o}$ is the characteristic turnover mass above which the metallicity asymptotically approaches the upper metallicity limit, $Z_{o}$. At stellar masses $<M_{o}$, the MZ relation reduces to a power law with an index $\gamma$.
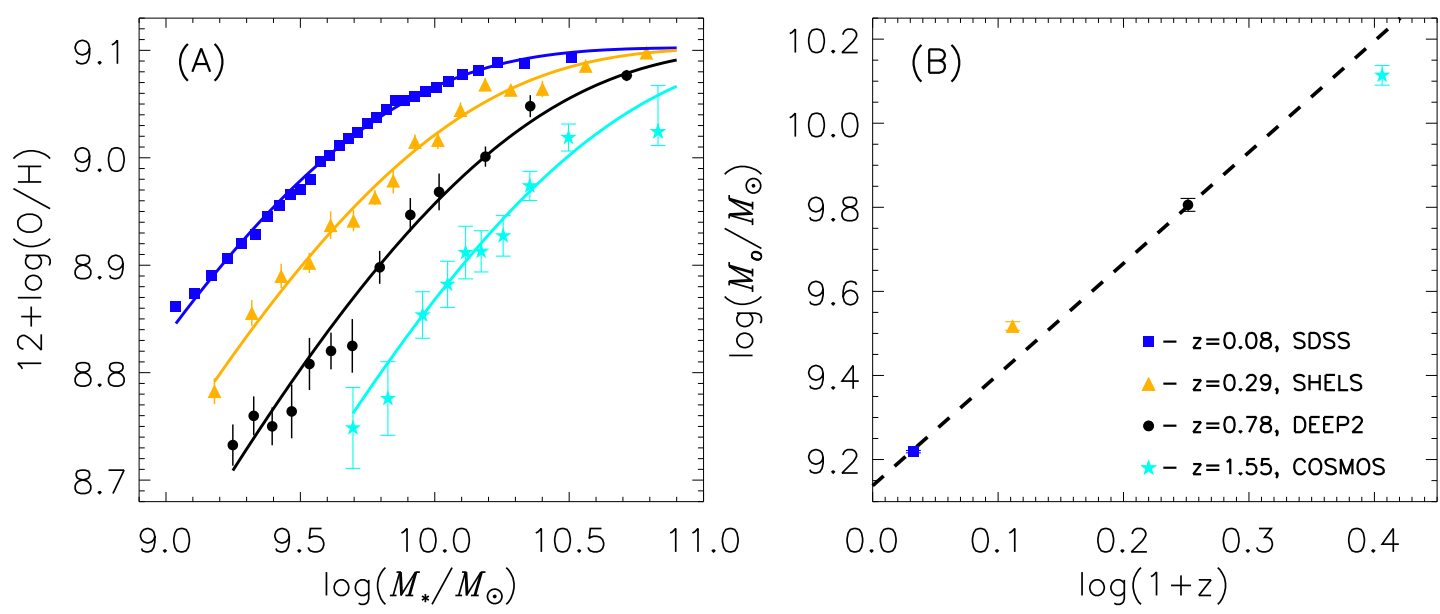

Figure 1: (A) The MZ relation derived from four galaxy samples ranging to $z=1.55$. The data are fit by the model parameterized by Equation 2.1. The fits are the single parameter model (see text). (B) $M_{o}$ as a function of redshift derived from fitting Equation 2.1 to the data and fixing the values of $Z_{o}$ and $\gamma$ to the best-fit parameters derived from the SDSS sample. Figure credit: Zahid et al. 2014. [40].

We plot the MZ relation for $z \lesssim 1.6$ in Figure $1 \mathrm{~A}$. The data show that at a fixed stellar mass, the metallicity increases as the Universe evolves. The MZ relation flattens at high stellar masses. Massive metal-rich galaxies at high redshifts have metallicities comparable to massive local galaxies. In Zahid et al. [17] we show the flattening of the MZ relation results from galaxies enriching to an empirical metallicity limit. This limit is parameterized by $Z_{o}$ and does not evolve significantly with redshift. The solid curves in Figure 1A show the single parameter model fits. The redshift evolution of the MZ relation can be parameterized solely by evolution in the characteristic turnover mass. The value of $M_{o}$ derived from fitting the single parameter model is plotted in Figure 1B and the fit of the data is

$$
\log \left(M_{o} / M_{\odot}\right)=(9.138 \pm 0.003)+(2.64 \pm 0.05) \log (1+z)
$$

The single parameter model fit to the data shows that the characteristic turnover mass, $M_{o}$, is an order of magnitude larger at $z \sim 1.6$.

The data are consistent with a single, redshift independent value for $Z_{o}$ and $\gamma$. The fact that the MZ relation evolution only depends on the characteristic turnover mass, $M_{o}$, means that relation between metallicity and stellar mass scaled by $M_{o}$ is independent of redshift. We plot the relation between metallicity and $\gamma \log \left(M_{*} / M_{o}\right)$ in Figure 2. The key to unlocking the origin of the MZ 


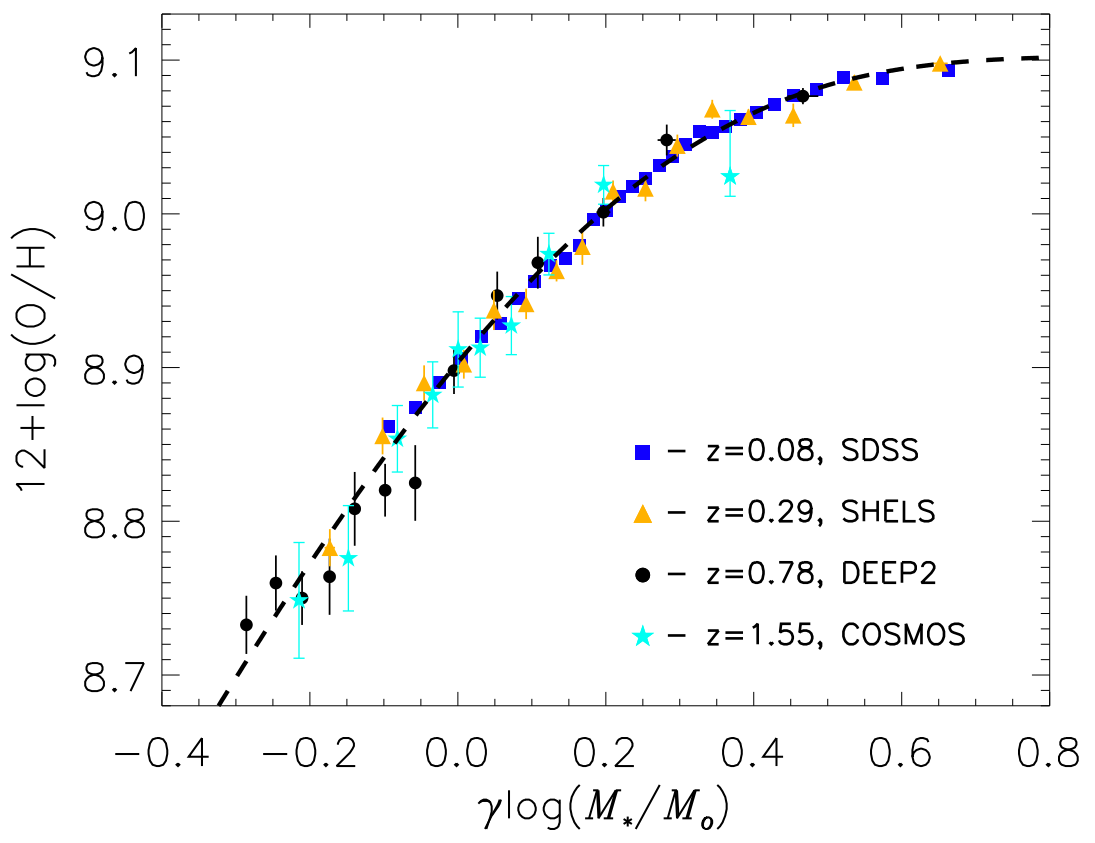

Figure 2: The metallicity plotted against the stellar mass normalized to the measured characteristic turnover mass, $M_{o}$ of each data set. Figure credit: Zahid et al. 2014. [40].

relation is our ability to explain the relation plotted in Figure 2. Thus we must determine physical meaning of $Z_{o}, \gamma$ and $M_{o}$.

\section{Analytical Solutions to the Equations of Galactic Chemical Evolution}

We begin our exploration of the physical origin of the MZ relation by examining analytical models of chemical evolution. The equations below do not necessarily have analytical solutions. We will make several physically motivated simplifying assumptions to arrive at an analytical solution. The model we derive below is the inflow model first introduced by Larson [42]. The novel aspect of our model is our treatment of the impact of outflows.

While metallicities are traditionally quoted as a number density of oxygen to hydrogen, in the following discussion we define the metallicity as $Z \equiv M_{z} / M_{g}$. Here $M_{z}$ is the mass of oxygen in the gas-phase and $M_{g}$ is the hydrogen gas mass. A constant scale factor relates metallicity defined in terms of mass density to metallicity defined in terms of number density [43]. We analyze the case of a single galaxy as it evolves chemically. We can thus parameterize the problem only in terms of stellar mass. To model the chemical evolution of arbitrary galaxy populations, time must be included in the equations. In this case, partial derivatives replace all derivatives below. The analytical solution that we derive based on a single galaxy is generalizable to the case of an arbitrary galaxy population. Our interpretation is independent of whether we analyze the case of a single galaxy or a population of galaxies. 
We start with the equation of chemical evolution describing the change in the metallicity with respect to stellar mass. This is given by

$$
\frac{\mathrm{d} Z}{\mathrm{~d} M_{*}}=\frac{\mathrm{d}}{\mathrm{d} M_{*}}\left(\frac{M_{z}}{M_{g}}\right)=\frac{1}{M_{g}}\left(\frac{\mathrm{d} M_{z}}{\mathrm{~d} M_{*}}-Z \frac{\mathrm{d} M_{g}}{\mathrm{~d} M_{*}}\right) .
$$

From observations we know that at low stellar masses we have an MZ relation with a positive power law index. In this case we necessarily have

$$
\frac{\mathrm{d} M_{z}}{\mathrm{~d} M_{*}}>Z \frac{\mathrm{d} M_{g}}{\mathrm{~d} M_{*}}
$$

When the metallicity is small, the second term of Equation $3.1\left(Z \frac{\mathrm{d} M_{g}}{\mathrm{~d} M_{*}}\right)$ is negligible. From measurements of the stellar and gas content of galaxies in the local Universe, we have that $M_{g} \propto \sqrt{M_{*}}$ $[44,45]$. The change in gas mass with respect to stellar mass is then $\mathrm{d} M_{g} / \mathrm{d} M_{*} \propto 1 / \sqrt{M_{*}}$. At higher stellar masses, the second term of the right hand side of Equation $3.1\left(Z \frac{\mathrm{d} M_{g}}{\mathrm{~d} M_{*}}\right)$ tends to zero. In this case, the change in metallicity with respect to stellar mass can be approximated by

$$
\frac{\mathrm{d} Z}{\mathrm{~d} M_{*}} \approx \frac{1}{M_{g}} \frac{\mathrm{d} M_{z}}{\mathrm{~d} M_{*}}
$$

This equation simply states that the chemical evolution of a galaxy is dominated by the production of metals and not by a slowly changing gas reservoir.

To solve Equation 3.3 we must define the right hand side. The change in oxygen mass is given by

$$
\mathrm{d} M_{z}=Y \mathrm{~d} M_{*}-Z \mathrm{~d} M_{*}+R Z \mathrm{~d} M_{*}+Z_{i} \mathrm{~d} M_{i}-Z_{w} \mathrm{~d} M_{w} .
$$

The first term on the right hand side is the production of newly synthesized oxygen where $Y$ is the nucleosynthetic yield. $Y$ is the mass of oxygen created per unit SFR. $\mathrm{d} M_{*}$ is the mass of newly formed stars, i.e. the SFR. $Y$ does not depend strongly on any galaxy properties [46, 47]. We treat $Y$ as a constant. The second term on the right hand side is the mass of oxygen in the ISM that forms into stars. The second term is negative indicating that the oxygen going into stars is taken out of the ISM. The third term on the right hand side is the mass of oxygen that goes into stars but is returned back to the ISM via stellar mass loss where $R$ is the fraction of mass returned back to the ISM. The third term represents previously synthesized oxygen returned back to the ISM by stellar winds and supernovae. The timescale for stellar mass return is short compared to the galaxy evolution timescale. We assume that stellar mass is instantaneously returned back into the ISM. The fourth and fifth terms represent the inflows and outflows (winds) of oxygen, respectively. The terms $Z_{i}, \mathrm{~d} M_{i}, Z_{w}$ and $\mathrm{d} M_{w}$ are the inflow metallicity, mass rate of inflow, outflow metallicity and mass rate of outflows, respectively. Dividing Equation 3.4 by $\mathrm{d} M_{*}$ we obtain

$$
\frac{\mathrm{d} M_{z}}{\mathrm{~d} M_{*}}=Y-Z(1-R)+Z_{i} \frac{\mathrm{d} M_{i}}{\mathrm{~d} M_{*}}-Z_{w} \frac{\mathrm{d} M_{w}}{\mathrm{~d} M_{*}} .
$$

This equation is the rate of change of the oxygen mass with respect to the SFR.

We combine the effects of outflows and inflows into a single factor defined as

$$
\zeta \equiv Z_{w} \frac{\mathrm{d} M_{w}}{\mathrm{~d} M_{*}}-Z_{i} \frac{\mathrm{d} M_{i}}{\mathrm{~d} M_{*}} .
$$


$\zeta$ is the oxygen mass loss factor. Each of the terms on the right hand side of Equation 3.6 are uncertain [48]. However, in Zahid et al. [16] we derive empirical constraints for the net loss of oxygen from galaxies. We estimate the total mass of oxygen produced by galaxies from their current stellar mass. We estimate the mass of oxygen in the gas-phase from the MZ relation and the scaling between stellar mass and gas mass observed in local galaxies. We empirically constrain the mass of oxygen locked up in stars by constructing a self-consistent empirical model based on multi-epoch observations of SFRs and metallicities of galaxies. Based on these estimates, we show that the total mass of oxygen expelled from galaxies over their lifetime is nearly proportional to their stellar mass. This means

$$
\int_{0}^{M_{*}} \zeta \mathrm{d} M_{*}^{\prime} \propto M_{*}
$$

implying that $\zeta$ is constant. In a more recent empirical analysis, Peeples et al. [45] confirm that the mass of oxygen lost relative to the mass of oxygen produced is independent of stellar mass in starforming galaxies. They estimate that on average, galaxies lose $\sim 80 \%$ of the oxygen they produce. The physical mechanism of outflows is not well understood though it is generally considered to be driven by energy/momentum from massive stars. Perhaps it is not that surprising that the mass of oxygen expelled from a galaxy scales with stellar mass since the total energy/momentum which is responsible for driving outflows in a galaxy is directly proportional to current stellar mass. With the adoption of $\zeta$ as a constant we have

$$
\frac{\mathrm{d} Z}{\mathrm{~d} M_{*}} \approx \frac{Y_{N}-Z(1-R)}{M_{g}}
$$

Here we have combined the production and loss of oxygen, both of which are proportional to stellar mass, into a single term defined as $Y_{N} \equiv Y-\zeta$. We refer to this as the net yield. The net yield is the mass of oxygen produced by star formation modulo the oxygen expelled from the ISM.

The measured hydrogen gas mass of star forming galaxies is reasonably well described by a power law over $\sim 4$ decades in stellar mass $[44,45]$. To solve Equation 3.8 we parameterize the relation between gas mass and stellar mass by

$$
M_{g}=G M_{*}^{g},
$$

where $G$ is the zero point and $g$ is the power law index of the relation. The solution to Equation 3.8 is

$$
Z\left(M_{*}\right)=\frac{Y_{N}}{1-R}\left[1-\exp \left(-\left[\frac{1-R}{1-g}\right] \frac{M_{*}}{M_{g}}\right)\right] .
$$

This equation has the same form as the equation we fit to the MZ relation (Equation 2.1). By taking the logarithm of Equation 3.10, we can directly relate our fit parameters in Equation 2.1 to the physical parameters analytically describing chemical evolution. The asymptotic metallicity $Z_{o}$ is

$$
Z_{o}=\log \left(\frac{Y_{N}}{1-R}\right)
$$

The maximum metallicity observed in galaxies is set by the net yield, $Y_{N}$. From Equation 3.8 we see that the metallicity saturates $\left(\mathrm{d} Z / \mathrm{d} M_{*} \approx 0\right)$ when the amount of metals produced, $Y_{N}$, equals the 
amount of metals locked up in stars, $Z(1-R)$. The arguments of the exponentials in Equations 2.1 and 3.10 can be equated to give

$$
\frac{1-R}{1-g}\left(\frac{M_{*}}{M_{g}}\right) \approx \frac{M_{*}}{M_{g}} \approx\left(\frac{M_{*}}{M_{o}}\right)^{\gamma} .
$$

The return fraction $R$ and the power law index of the gas mass relation, $g$, are nearly equal $(R \sim$ $g \sim 0.5$ ). Hereafter, we drop the prefactor term of the left hand side of Equation 3.12. Substituting our relation for the gas mass as a function of stellar mass from Equation 3.9 we have

$$
\left(\frac{M_{*}}{M_{o}}\right)^{\gamma} \approx \frac{M_{*}^{1-g}}{G}
$$

The low mass end slope we fit to the MZ relation, $\gamma$, is related to the power law index of the gas mass relation by $\gamma=1-g$. The characteristic turnover mass, $M_{o}$, is related to the zero point of the relation between gas mass and stellar mass by $M_{o}=G^{1 / \gamma} . M_{o}$ is the stellar mass at which the gas-to-stellar mass ratio is unity. This interpretation suggests that the redshift evolution of the MZ relation is due to the larger gas masses of galaxies at early times. In Figure 2 we show a redshift independent relation between metallicity and $\left(M_{*} / M_{o}\right)^{\gamma}$. From examination of Equation 3.12, we learn that $\left(M_{*} / M_{o}\right)^{\gamma}=M_{*} / M_{g}$. Thus, the redshift independent relation plotted in Figure 2 is a relation between metallicity and stellar-to-gas mass ratio.

\section{The Universal Metallicity Relation: The Origin of the Mass-Metallicity Relation and its Evolution}

We analyze observations of the MZ relation for $z \lesssim 1.6$. The MZ relation exhibits very simple redshift evolution which we parameterize by redshift evolution in the characteristic turnover mass where the metallicity begins to saturate. We physically interpret our fit parameters by comparing them to solutions of analytical models of chemical evolution. Our analysis provides a simple and intuitive perspective of galactic chemical evolution. Galaxies follow a universal relation between metallicity and stellar-to-gas mass ratio as they evolve. Metallicity is defined as the ratio of oxygen to hydrogen. The net mass of oxygen produced is directly proportional to galaxy stellar mass. Given the definition of metallicity, a universal relation between metallicity and stellar-to-gas mass ratio should be expected.

The chemical evolution of galaxies can be characterized as having three distinct regimes: gasrich, gas-poor and gas-depleted. Figure 3 is a schematic which illustrates the three regimes. The gas-rich regime is plotted in blue. Galaxies are in the gas-rich regime when $M_{g}>M_{*}$. In the gas-rich regime, the metallicity is proportional to stellar-to-gas mass ratio. This can be seen by Taylor expanding Equation 3.10. The gas-poor regime is plotted in green. Galaxies cross over to the gas-poor regime when $M_{g}<M_{*}$. In the gas-poor regime the metallicity is high enough that the mass of oxygen that is being locked up in stars becomes an appreciable fraction of the mass of oxygen produced. Galaxies exponentially approach the metallicity saturation limit. The gasdepleted regime is plotted in red. Galaxies in the gas-depleted regime have $M_{g} / M_{*} \ll 1$. In this

regime, the metallicity is so high that the mass of oxygen taken out of the ISM and locked up in low 


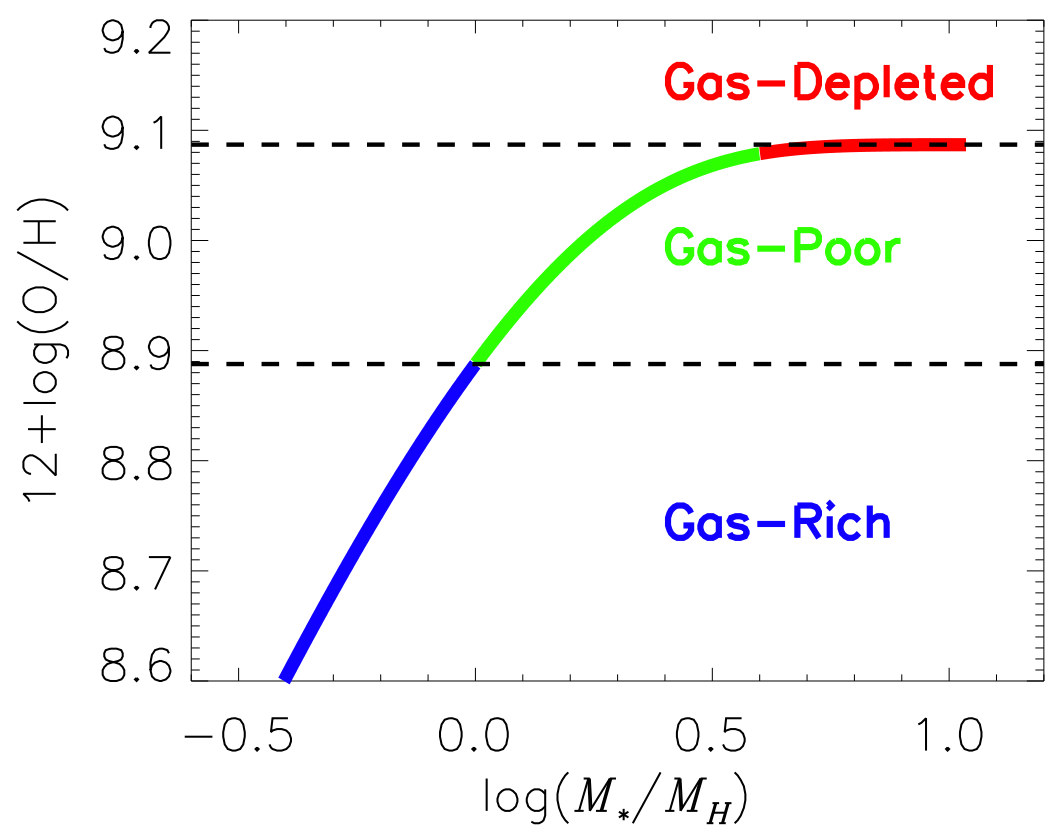

Figure 3: A schematic illustrating the three regimes of galactic chemical evolution.

mass stars equals the mass of oxygen produced by massive stars. The metallicity can not increase beyond this point.

The MZ relation originates from the more fundamental universal relation between metallicity and stellar-to-gas mass ratio. At a fixed stellar mass, the metallicities of galaxies increase as the Universe evolves because of a commensurate decline in their gas content. The slope of the MZ relation at $M_{*}<M_{o}$ is set by the slope of the relation between gas mass and stellar mass. This is because the slope of the more fundamental relation between metallicity and gas-to-stellar mass ratio is unity for galaxies with $M_{*}<M_{o}$ (see Equation 3.10). The scatter observed in the MZ relation largely reflects the scatter in gas mass at a fixed stellar mass. The relation between stellar mass, metallicity and SFR [23] is a natural consequence of the universal metallicity relation. At a fixed stellar mass, galaxies with larger gas reservoirs will typically exhibit elevated SFRs and diluted metallicities and vice versa. Several authors have recognized variations in the gas content of galaxies as the basis of the observed relation between stellar mass, metallicity and SFR [49, 50, $33,51,52]$. Our results are consistent with this interpretation.

The universal relation of chemical evolution implies that the metallicity of a galaxy is instantaneously set by its stellar-to-gas mass ratio. Gas flows and star formation move galaxies along - not off-the universal metallicity relation. Unlike the $\mathrm{MZ}$ relation in which the stellar mass is monotonically increasing, the stellar-to-gas mass ratio can increase or decrease. The response of galaxies to large gas accretion events will be to instantaneously move down the universal metallicity relation. Conversely, galaxies will move up the universal metallicity relation as they deplete their gas reservoirs by star formation and outflows. 
The intrinsic scatter in the universal metallicity relation is likely to be small. At a fixed stellar mass we expect some scatter in the net yield, $Y_{N}$ due to scatter in total mass of oxygen expelled from galaxies. Any scatter in the net yield would directly translate into scatter in the universal metallicity relation. Mergers and starbursts will likely also contribute to the scatter in the universal metallicity relation since these events significantly disrupt the ISM of galaxies. The observed scatter in the FMR of Mannucci et al. [23] and HI FMR of Bothwell et al. [33] is $~ 0.07$ dex. Since the FMR is directly a result of the universal metallicity relation, the measured scatter in the FMR provides an upper limit for the scatter in the universal metallicity relation.

If our interpretation is correct, the metallicities of galaxies provide a precise probe of their ISM gas content. We derive the relation between average gas mass and stellar mass by combining Equations 2.2 and 3.12. The average mass of hydrogen gas in the star-forming disk of galaxies as a function of redshift and stellar mass is

$$
M_{g}\left(M_{*}, z\right)=3.87 \times 10^{9}(1+z)^{1.35}\left(\frac{M_{*}}{10^{10} M_{\odot}}\right)^{0.49}\left[M_{\odot}\right] .
$$

In the absence of direct measurements, the gas mass of galaxies can be estimated from measurements of stellar mass and metallicity by combining Equation 2.1 and 3.12 and solving for $M_{g}$. The hydrogen gas mass as a function of stellar mass and metallicity is

$$
M_{g}\left(M_{*}, Z\right)=\frac{-M_{*}}{\ln \left(1-10^{\left[Z-Z_{o}\right]}\right)}\left[M_{\odot}\right]
$$

The stellar mass is measured in units of $M_{\odot}$ and $Z_{o}=9.10$. We emphasize that metallicities must be converted into the KK04 diagnostic when using Equation 4.2. Gas masses estimated using Equation 4.2 carry the uncertainties associated with stellar masses, metallicities and the intrinsic scatter in the universal metallicity relation. The KK04 strong-line metallicity method is estimated to have an accuracy of $\sim 0.15 \mathrm{dex}$ [53] and we estimate that stellar masses are accurate to $\sim 0.1$ dex once systematic differences between methods are removed (see Section 3.1). Combining these uncertainties with the $\lesssim 0.07$ dex estimated scatter in the universal metallicity relation, we conclude that the gas masses estimated using Equation 4.2 are accurate to within $\sim 0.3$ dex. The relations presented by Equation 4.1 and 4.2 are valid for galaxies at $z \lesssim 1.6$. These relations are testable predictions of the gas content of galaxies based on the observed evolution of the MZ relation. However, we note that in the saturation regime, the metallicity is not sensitive to the stellar-to-gas mass ratio.

We have provided evidence for a universal relation between metallicity and stellar-to-gas mass ratio for galaxies at $z \lesssim 1.6$. Our observations only extend down to stellar masses of $\sim 10^{9} M_{\odot}$ and gas-to-stellar mass ratios of $\sim 0.5$. By summing the spectra of $\sim 200,000$ galaxies in the SDSS, Andrews \& Martini [6] measure the MZ relation down to stellar masses of $\sim 10^{7.5} M_{\odot}$. They measure a continuous MZ relation which flattens at high stellar masses and scales as $O / H \propto M_{*}^{1 / 2}$ at stellar masses $<10^{9} M_{\odot}$. Our measurements are consistent with the scaling of the MZ relation measured by Andrews \& Martini [6] suggesting that the universal metallicity relation may extend well into the dwarf regime.

The physical basis of the universal metallicity relation needs to be explored in much greater detail. Several aspects of this work imply a cosmological origin for the relation. The evolution 
of the characteristic turnover mass, $M_{o}$, is proportional to $(1+z)^{2.6}$. This scaling with redshift is similar to the $(1+z)^{2.5}$ scaling of the growth rate of dark matter halos in simulations [54]. Additionally, our observations suggest that $M_{g} \propto \sqrt{M_{*}}$ for $z \lesssim 1.6$. This scaling of gas and stellar mass is similar to the scaling between stellar and halo mass, $M_{h}$, determined from abundance matching [55] and from galaxy-galaxy weak lensing, galaxy clustering and galaxy distribution [56]. This implies that $M_{g} / M_{h}$ is nearly constant with respect to stellar mass. The slope of the relation between stellar mass and halo mass does not evolve significantly out to $z \sim 1.6[56,55]$. An $M_{g} / M_{h}$ ratio that is constant with stellar mass may be the physical origin for the constant slope of the relation between gas mass and stellar mass.

A detailed analysis of systematic issues related to measurements of the MZ relation should soon be possible as larger data sets become available. In particular, metallicities are derived using techniques that are calibrated using observations of local galaxies. If ISM conditions evolve with redshift, these calibrations may need to be revised. Our analysis is primarily based on the R23 diagnostic calibrated by Kobulnicky \& Kewley [57]. However, long-standing discrepancies between various metallicity diagnostics remain unresolved [58, 59, 60, 61, 62]. Discrepancies between various metallicity diagnostics do not change the major conclusions of this work but the quantitative analysis presented is subject to these uncertainties. Additionally, larger and more complete spectroscopic samples combined with cosmological simulations should allow us to assess selection biases in our measurement of the MZ relation. The MZ relation appears to be insensitive to selection biases and we anticipate that correction for these biases will not change the major conclusions presented in this work.

\section{References}

[1] J. Lequeux, M. Peimbert, J. F. Rayo, A. Serrano, and S. Torres-Peimbert, Chemical composition and evolution of irregular and blue compact galaxies, $A \& A 80$ (Dec., 1979) 155-166.

[2] C. A. Tremonti, T. M. Heckman, G. Kauffmann, J. Brinchmann, S. Charlot, S. D. M. White, M. Seibert, E. W. Peng, D. J. Schlegel, A. Uomoto, M. Fukugita, and J. Brinkmann, The Origin of the Mass-Metallicity Relation: Insights from 53,000 Star-forming Galaxies in the Sloan Digital Sky Survey, ApJ 613 (Oct., 2004) 898-913, [ast ro-ph / 0].

[3] H. Lee, E. D. Skillman, J. M. Cannon, D. C. Jackson, R. D. Gehrz, E. F. Polomski, and C. E. Woodward, On Extending the Mass-Metallicity Relation of Galaxies by 2.5 Decades in Stellar Mass, ApJ 647 (Aug., 2006) 970-983, [astro-ph / 0].

[4] D. A. Berg, E. D. Skillman, A. R. Marble, L. van Zee, C. W. Engelbracht, J. C. Lee, R. C. Kennicutt, Jr., D. Calzetti, D. A. Dale, and B. D. Johnson, Direct Oxygen Abundances for Low-luminosity LVL Galaxies, ApJ 754 (Aug., 2012) 98, [arXiv: 1205.6782].

[5] H. J. Zahid, F. Bresolin, L. J. Kewley, A. L. Coil, and R. Davé, The Metallicities of Low Stellar Mass Galaxies and the Scatter in the Mass-Metallicity Relation, ApJ 750 (May, 2012) 120, [arXiv: 1203.0558].

[6] B. H. Andrews and P. Martini, The Mass-Metallicity Relation with the Direct Method on Stacked Spectra of SDSS Galaxies, ApJ 765 (Mar., 2013) 140, [arXiv: 1211.3418].

[7] S. Savaglio, K. Glazebrook, D. Le Borgne, S. Juneau, R. G. Abraham, H.-W. Chen, D. Crampton, P. J. McCarthy, R. G. Carlberg, R. O. Marzke, K. Roth, I. Jørgensen, and R. Murowinski, The Gemini 
Deep Deep Survey. VII. The Redshift Evolution of the Mass-Metallicity Relation, ApJ 635 (Dec., 2005) 260-279, [astro-ph / 0].

[8] D. K. Erb, A. E. Shapley, M. Pettini, C. C. Steidel, N. A. Reddy, and K. L. Adelberger, The Mass-Metallicity Relation at z> 2, ApJ 644 (June, 2006) 813-828, [astro-ph / 0].

[9] C. Maier, S. J. Lilly, C. M. Carollo, A. Stockton, and M. Brodwin, Near-Infrared Spectroscopy of 0.4 $<z<1.0$ CFRS Galaxies: Oxygen Abundances, SFRs, and Dust, ApJ 634 (Dec., 2005) 849-860, [astro-ph/0].

[10] C. Maier, S. J. Lilly, C. M. Carollo, K. Meisenheimer, H. Hippelein, and A. Stockton, Oxygen Gas Abundances at z 1.4: Implications for the Chemical Evolution History of Galaxies, ApJ 639 (Mar., 2006) 858-867, [astro-ph / 0 ].

[11] L. L. Cowie and A. J. Barger, An Integrated Picture of Star Formation, Metallicity Evolution, and Galactic Stellar Mass Assembly, ApJ 686 (Oct., 2008) 72-116, [arXiv : 0806 . 3457].

[12] R. Maiolino, T. Nagao, A. Grazian, F. Cocchia, A. Marconi, F. Mannucci, A. Cimatti, A. Pipino, S. Ballero, F. Calura, C. Chiappini, A. Fontana, G. L. Granato, F. Matteucci, G. Pastorini, L. Pentericci, G. Risaliti, M. Salvati, and L. Silva, AMAZE. I. The evolution of the mass-metallicity relation at $z>3, A \& A 488$ (Sept., 2008) 463-479, [arXiv : 0806.2410 ].

[13] F. Mannucci, G. Cresci, R. Maiolino, A. Marconi, G. Pastorini, L. Pozzetti, A. Gnerucci, G. Risaliti, R. Schneider, M. Lehnert, and M. Salvati, LSD: Lyman-break galaxies Stellar populations and Dynamics - I. Mass, metallicity and gas at z 3.1, MNRAS 398 (Oct., 2009) 1915-1931, [arXiv:0902.2398].

[14] H. J. Zahid, L. J. Kewley, and F. Bresolin, The Mass-Metallicity and Luminosity-Metallicity Relations from DEEP2 at z 0.8, ApJ 730 (Apr., 2011) 137-+, [arXiv: 1006.4877$].$

[15] K. Yabe, K. Ohta, F. Iwamuro, S. Yuma, M. Akiyama, N. Tamura, M. Kimura, N. Takato, Y. Moritani, M. Sumiyoshi, T. Maihara, J. Silverman, G. Dalton, I. Lewis, D. Bonfield, H. Lee, E. Curtis Lake, E. Macaulay, and F. Clarke, NIR Spectroscopy of Star-Forming Galaxies at $z \sim 1.4$ with Subaru/FMOS: The Mass-Metallicity Relation, PASJ 64 (June, 2012) 60, [arXiv:1112 . 3704 ].

[16] H. J. Zahid, G. I. Dima, L. J. Kewley, D. K. Erb, and R. Davé, A Census of Oxygen in Star-forming Galaxies: An Empirical Model Linking Metallicities, Star Formation Rates, and Outflows, ApJ 757 (Sept., 2012) 54, [arXiv: 1207.5509 ].

[17] H. J. Zahid, M. J. Geller, L. J. Kewley, H. S. Hwang, D. G. Fabricant, and M. J. Kurtz, The Chemical Evolution of Star-forming Galaxies over the Last 11 Billion Years, ApJ 771 (July, 2013) L19, [arXiv:1303.5987].

[18] H. J. Zahid, D. Kashino, J. D. Silverman, L. J. Kewley, E. Daddi, A. Renzini, G. Rodighiero, T. Nagao, N. Arimoto, D. B. Sanders, J. Kartaltepe, S. J. Lilly, C. Maier, M. J. Geller, P. Capak, C. M. Carollo, J. Chu, G. Hasinger, O. Ilbert, M. Kajisawa, A. M. Koekemoer, K. Kovac\#728, O. Le Fèvre, D. Masters, H. J. McCracken, M. Onodera, N. Scoville, V. Strazzullo, N. Sugiyama, Y. Taniguchi, and The COSMOS Team, The FMOS-COSMOS Survey of Star-forming Galaxies at z 1.6. II. The Mass-Metallicity Relation and the Dependence on Star Formation Rate and Dust Extinction, ApJ 792 (Sept., 2014) 75, [arXiv:1310.4950].

[19] K. Yabe, K. Ohta, F. Iwamuro, M. Akiyama, N. Tamura, S. Yuma, M. Kimura, N. Takato, Y. Moritani, M. Sumiyoshi, T. Maihara, J. Silverman, G. Dalton, I. Lewis, D. Bonfield, H. Lee, E. Curtis-Lake, E. Macaulay, and F. Clarke, The mass-metallicity relation at $z \sim 1.4$ revealed with Subaru/FMOS, MNRAS 437 (Feb., 2014) 3647-3663, [arXiv:1311.2624]. 
[20] T. Laskar, E. Berger, and R.-R. Chary, Exploring the Galaxy Mass-metallicity Relation at z 3-5, ApJ 739 (Sept., 2011) 1, [arXiv:1102.1019].

[21] P. Møller, J. P. U. Fynbo, C. Ledoux, and K. K. Nilsson, Mass-metallicity relation from $z=5$ to the present: evidence for a transition in the mode of galaxy growth at $z=2.6$ due to the end of sustained primordial gas infall, MNRAS 430 (Apr., 2013) 2680-2687, [arXiv: 1301 . 5013].

[22] S. L. Ellison, D. R. Patton, L. Simard, and A. W. McConnachie, Clues to the Origin of the Mass-Metallicity Relation: Dependence on Star Formation Rate and Galaxy Size, ApJ 672 (Jan., 2008) L107-L110, [arXiv: 0711 . 4833].

[23] F. Mannucci, G. Cresci, R. Maiolino, A. Marconi, and A. Gnerucci, A fundamental relation between mass, star formation rate and metallicity in local and high-redshift galaxies, MNRAS 408 (Nov., 2010) 2115-2127, [arXiv: 1005.0006 ].

[24] M. A. Lara-López, J. Cepa, A. Bongiovanni, A. M. Pérez García, A. Ederoclite, H. Castañeda, M. Fernández Lorenzo, M. Pović, and M. Sánchez-Portal, A fundamental plane for field star-forming galaxies, A\&A $\mathbf{5 2 1}$ (Oct., 2010) L53+, [arXiv: 1005 . 0509].

[25] R. M. Yates, G. Kauffmann, and Q. Guo, The relation between metallicity, stellar mass and star formation in galaxies: an analysis of observational and model data, MNRAS 422 (May, 2012) 215-231, [arXiv:1107.3145].

[26] P. Troncoso, R. Maiolino, V. Sommariva, G. Cresci, F. Mannucci, A. Marconi, M. Meneghetti, A. Grazian, A. Cimatti, A. Fontana, T. Nagao, and L. Pentericci, Metallicity evolution, metallicity gradients, and gas fractions at z 3.4, A\&A $\mathbf{5 6 3}$ (Mar., 2014) A58, [arXiv:1311.4576].

[27] Y. Niino, The Redshift Evolution of the Relation between Stellar Mass, Star Formation Rate, and Gas Metallicity of Galaxies, ApJ 761 (Dec., 2012) 126, [arXiv:1205.1897].

[28] E. Pérez-Montero, T. Contini, F. Lamareille, C. Maier, C. M. Carollo, J.-P. Kneib, O. Le Fèvre, S. Lilly, V. Mainieri, A. Renzini, M. Scodeggio, G. Zamorani, S. Bardelli, M. Bolzonella, A. Bongiorno, K. Caputi, O. Cucciati, S. de la Torre, L. de Ravel, P. Franzetti, B. Garilli, A. Iovino, P. Kampczyk, C. Knobel, K. Kovač, J.-F. Le Borgne, V. Le Brun, M. Mignoli, R. Pellò, Y. Peng, V. Presotto, E. Ricciardelli, J. D. Silverman, M. Tanaka, L. A. M. Tasca, L. Tresse, D. Vergani, and E. Zucca, The cosmic evolution of oxygen and nitrogen abundances in star-forming galaxies over the past 10 Gyr, A\&A 549 (Jan., 2013) A25, [arXiv:1210.0334].

[29] S. F. Sánchez, F. F. Rosales-Ortega, B. Jungwiert, J. Iglesias-Páramo, J. M. Vílchez, R. A. Marino, C. J. Walcher, B. Husemann, D. Mast, A. Monreal-Ibero, R. Cid Fernandes, E. Pérez, R. González Delgado, R. García-Benito, L. Galbany, G. van de Ven, K. Jahnke, H. Flores, J. Bland-Hawthorn, A. R. López-Sánchez, V. Stanishev, D. Miralles-Caballero, A. I. Díaz, P. Sánchez-Blazquez, M. Mollá, A. Gallazzi, P. Papaderos, J. M. Gomes, N. Gruel, I. Pérez, T. Ruiz-Lara, E. Florido, A. de Lorenzo-Cáceres, J. Mendez-Abreu, C. Kehrig, M. M. Roth, B. Ziegler, J. Alves, L. Wisotzki, D. Kupko, A. Quirrenbach, D. Bomans, and Califa Collaboration, Mass-metallicity relation explored with CALIFA. I. Is there a dependence on the star-formation rate?, A\&A 554 (June, 2013) A58, [arXiv:1304.2158].

[30] C. Ly, M. A. Malkan, T. Nagao, N. Kashikawa, K. Shimasaku, and M. Hayashi, ”Direct” Gas-phase Metallicities, Stellar Properties, and Local Environments of Emission-line Galaxies at Redshifts below 0.90, ApJ 780 (Jan., 2014) 122, [arXiv: 1307. 7712].

[31] T. M. Hughes, L. Cortese, A. Boselli, G. Gavazzi, and J. I. Davies, The role of cold gas and environment on the stellar mass-metallicity relation of nearby galaxies, A\&A $\mathbf{5 5 0}$ (Feb., 2013) A115, [arXiv:1207.4191]. 
[32] M. A. Lara-López, A. M. Hopkins, A. R. López-Sánchez, S. Brough, M. Colless, J. Bland-Hawthorn, S. Driver, C. Foster, J. Liske, J. Loveday, A. S. G. Robotham, R. G. Sharp, O. Steele, and E. N. Taylor, Galaxy And Mass Assembly (GAMA): the connection between metals, specific SFR and H I gas in galaxies: the Z-SSFR relation, MNRAS 433 (June, 2013) L35-L39, [arXiv: 1304 . 3889].

[33] M. S. Bothwell, R. Maiolino, R. Kennicutt, G. Cresci, F. Mannucci, A. Marconi, and C. Cicone, A fundamental relation between the metallicity, gas content and stellar mass of local galaxies, MNRAS 433 (Aug., 2013) 1425-1435, [arXiv: 1304 . 4940].

[34] R. B. Larson, Effects of supernovac on the early evolution of galaxies, MNRAS 169 (Nov., 1974) 229-246.

[35] A. M. Brooks, F. Governato, C. M. Booth, B. Willman, J. P. Gardner, J. Wadsley, G. Stinson, and T. Quinn, The Origin and Evolution of the Mass-Metallicity Relationship for Galaxies: Results from Cosmological N-Body Simulations, ApJ 655 (Jan., 2007) L17-L20, [astro-ph / 0].

[36] K. Finlator and R. Davé, The origin of the galaxy mass-metallicity relation and implications for galactic outflows, MNRAS 385 (Apr., 2008) 2181-2204, [arXiv: 0704 . 3100].

[37] F. Calura, A. Pipino, C. Chiappini, F. Matteucci, and R. Maiolino, The evolution of the mass-metallicity relation in galaxies of different morphological types, A\&A 504 (Sept., 2009) 373-388, [arXiv:0904.2180].

[38] J. J. Dalcanton, P. Yoachim, and R. A. Bernstein, The Formation of Dust Lanes: Implications for Galaxy Evolution, ApJ 608 (June, 2004) 189-207, [astro-ph / 0].

[39] J. Köppen, C. Weidner, and P. Kroupa, A possible origin of the mass-metallicity relation of galaxies, MNRAS 375 (Feb., 2007) 673-684, [astro-ph / 0].

[40] J. Zahid, G. Dima, R. Kudritzki, L. Kewley, M. Geller, and H. S. Hwang, The Universal Relation of Galactic Chemical Evolution: The Origin of the Mass-Metallicity Relation, ArXiv e-prints (Apr., 2014) [arXiv: 1404 . 7526].

[41] J. Moustakas, D. Zaritsky, M. Brown, R. Cool, A. Dey, D. J. Eisenstein, A. H. Gonzalez, B. Jannuzi, C. Jones, C. S. Kochanek, S. S. Murray, and V. Wild, Evolution of the Stellar Mass-Metallicity Relation Since $z=0.75$, ArXiv e-prints (Dec., 2011) [arXiv:1112.3300].

[42] R. B. Larson, Effect of Infalling Matter on the Heavy Element Content of a Galaxy, Nature Physical Science 236 (Mar., 1972) 7-8.

[43] M. S. Peeples and F. Shankar, Constraints on star formation driven galaxy winds from the mass-metallicity relation at $z=0$, MNRAS 417 (Nov., 2011) 2962-2981, [arXiv: 1007 . 3743].

[44] E. Papastergis, A. Cattaneo, S. Huang, R. Giovanelli, and M. P. Haynes, A Direct Measurement of the Baryonic Mass Function of Galaxies and Implications for the Galactic Baryon Fraction, ApJ $\mathbf{7 5 9}$ (Nov., 2012) 138, [arXiv: 1208 .5229].

[45] M. S. Peeples, J. K. Werk, J. Tumlinson, B. D. Oppenheimer, J. X. Prochaska, N. Katz, and D. H. Weinberg, A Budget and Accounting of Metals at z 0: Results from the COS-Halos Survey, ApJ 786 (May, 2014) 54, [arXiv: 1310.2253$].$

[46] D. Thomas, L. Greggio, and R. Bender, Stellar Yields and Chemical Evolution - I. Abundance Ratios and Delayed Mixing in the Solar Neighbourhood, MNRAS 296 (May, 1998) 119-149, [astro-ph/9].

[47] C. Kobayashi, H. Umeda, K. Nomoto, N. Tominaga, and T. Ohkubo, Galactic Chemical Evolution: Carbon through Zinc, ApJ 653 (Dec., 2006) 1145-1171, [astro-ph / 0]. 
[48] H. J. Zahid, P. Torrey, M. Vogelsberger, L. Hernquist, L. Kewley, and R. Davé, Empirical constraints for the magnitude and composition of galactic winds, Ap\&SS 349 (Feb., 2014) 873-879, [arXiv:1307.5909].

[49] R. Davé, K. Finlator, and B. D. Oppenheimer, An analytic model for the evolution of the stellar, gas and metal content of galaxies, MNRAS 421 (Mar., 2012) 98-107, [arXiv: 1108 . 0426].

[50] P. Dayal, A. Ferrara, and J. S. Dunlop, The physics of the fundamental metallicity relation, MNRAS 430 (Apr., 2013) 2891-2895, [arXiv: 1202 . 4770].

[51] S. J. Lilly, C. M. Carollo, A. Pipino, A. Renzini, and Y. Peng, Gas Regulation of Galaxies: The Evolution of the Cosmic Specific Star Formation Rate, the Metallicity-Mass-Star-formation Rate Relation, and the Stellar Content of Halos, ApJ 772 (Aug., 2013) 119, [arXiv : 1303.5059 ].

[52] R. Davé, N. Katz, B. D. Oppenheimer, J. A. Kollmeier, and D. H. Weinberg, The neutral hydrogen content of galaxies in cosmological hydrodynamic simulations, MNRAS 434 (Sept., 2013) 2645-2663, [arXiv:1302.3631].

[53] L. J. Kewley and S. L. Ellison, Metallicity Calibrations and the Mass-Metallicity Relation for Star-forming Galaxies, ApJ 681 (July, 2008) 1183-1204, [arXiv: 0801.1849 ].

[54] O. Fakhouri, C.-P. Ma, and M. Boylan-Kolchin, The merger rates and mass assembly histories of dark matter haloes in the two Millennium simulations, MNRAS 406 (Aug., 2010) 2267-2278, [arXiv:1001.2304].

[55] P. S. Behroozi, R. H. Wechsler, and C. Conroy, The Average Star Formation Histories of Galaxies in Dark Matter Halos from z = 0-8, ApJ 770 (June, 2013) 57, [arXiv: 1207.6105$].$

[56] A. Leauthaud, J. Tinker, K. Bundy, P. S. Behroozi, R. Massey, J. Rhodes, M. R. George, J.-P. Kneib, A. Benson, R. H. Wechsler, M. T. Busha, P. Capak, M. Cortês, O. Ilbert, A. M. Koekemoer, O. Le Fèvre, S. Lilly, H. J. McCracken, M. Salvato, T. Schrabback, N. Scoville, T. Smith, and J. E. Taylor, New Constraints on the Evolution of the Stellar-to-dark Matter Connection: A Combined Analysis of Galaxy-Galaxy Lensing, Clustering, and Stellar Mass Functions from $z=0.2$ to $z=1$, ApJ 744 (Jan., 2012) 159, [arXiv:1104.0928].

[57] H. A. Kobulnicky and L. J. Kewley, Metallicities of $0.3<z<1.0$ Galaxies in the GOODS-North Field, ApJ 617 (Dec., 2004) 240-261, [astro-ph/0].

[58] R.-P. Kudritzki, M. A. Urbaneja, F. Bresolin, N. Przybilla, W. Gieren, and G. Pietrzyński, Quantitative Spectroscopy of 24 A Supergiants in the Sculptor Galaxy NGC 300: Flux-weighted Gravity-Luminosity Relationship, Metallicity, and Metallicity Gradient, ApJ 681 (July, 2008) 269-289, [arXiv:0803.3654].

[59] F. Bresolin, W. Gieren, R.-P. Kudritzki, G. Pietrzyński, M. A. Urbaneja, and G. Carraro, Extragalactic Chemical Abundances: Do H II Regions and Young Stars Tell the Same Story? The Case of the Spiral Galaxy NGC 300, ApJ 700 (July, 2009) 309-330, [arXiv: 0905.2791$].$

[60] R.-P. Kudritzki, M. A. Urbaneja, Z. Gazak, F. Bresolin, N. Przybilla, W. Gieren, and G. Pietrzyński, Quantitative Spectroscopy of Blue Supergiant Stars in the Disk of M81: Metallicity, Metallicity Gradient, and Distance, ApJ 747 (Mar., 2012) 15, [arXiv:1112.3643].

[61] D. C. Nicholls, M. A. Dopita, and R. S. Sutherland, Resolving the Electron Temperature Discrepancies in H II Regions and Planetary Nebulae: $\kappa$-distributed Electrons, ApJ 752 (June, 2012) 148, [arXiv:1204.3880]. 
[62] M. A. Dopita, R. S. Sutherland, D. C. Nicholls, L. J. Kewley, and F. P. A. Vogt, New Strong-line Abundance Diagnostics for H II Regions: Effects of $\kappa$-distributed Electron Energies and New Atomic Data, ApJS 208 (Sept., 2013) 10, [arXiv: 1307.5950 ]. 